\title{
Lettre aux candidats à l'élection présidentielle
}

Madame, Monsieur,

La société moderne a connu un développement fulgurant grâce aux progrès des sciences et des technologies. Les problèmes rencontrés actuellement, tels la raréfaction des ressources ou le réchauffement climatique, ne pourront se résoudre que par la même voie, à savoir le développement de la recherche et de l'innovation. La France occupe encore une position enviable dans ce domaine et il importe de la maintenir, voire de la faire progresser.

Dans ce contexte, la Société Française de Physique est l'émanation d'une communauté nationale importante et de très haut niveau, comme en témoignent ses succès au niveau international. Toutefois, nos scientifiques sont légitimement inquiets de l'évolution de la politique de notre pays en matière de recherche et d'innovation. C'est pourquoi nous vous faisons parvenir un questionnaire destiné à nous éclairer. Nous souhaitons connaître vos réponses, pour les transmettre à l'ensemble de notre communauté via nos sites internet avant le vote du premier tour.

Voici la liste de nos questions, aussi précises que possible :

- Complexité du système de recherche : Celui-ci est formé de strates qui se sont accumulées ces dernières années, s'annihilant partiellement, favorisant l'immobilisme et nuisant à notre compétitivité. Quelle est votre vision finale du système simplifié ?

- Financement de la recherche : Il s'effectue de plus en plus par projets, souvent finalisés et dépendant de nombreux guichets. Ceci accroît énormément les tâches administratives dévolues aux chercheurs et nuit à la recherche fondamentale basée sur la créativité et la prise de risques. Que ferez-vous pour rétablir l'équilibre fondamentalappliqué ? En particulier, reviendrez-vous sur la politique de diminution des moyens récurrents tellement nécessaires à cet équilibre?

- Carrière des chercheurs : Le doctorat ès sciences est très mal reconnu en France. La carrière de chercheur ou d'enseignant-chercheur est mal rémunérée. Le doctorat est peu coté dans l'industrie, où il est barré par le diplôme d'Ingénieur, ce qui est préjudiciable au développement d'industries de pointe. Que comptez-vous faire pour remédier à cette situation typiquement française?

- Autonomie des Universités : Celle-ci s'est concrétisée récemment, avec diverses mesures censées renforcer leur rôle et leur compétitivité. Dans le même temps, le soutien accordé aux EPST tel le CNRS a été réduit de façon substantielle. Comptez-vous poursuivre cette tendance?

- Création d'emplois : Celle-ci dépend beaucoup du couplage entre recherche publique et privée, principalement avec les PME. Malgré les nombreuses officines nationales ou locales censées améliorer ce couplage, le résultat est décevant et bien inférieur à celui d'autres pays. Quelle sera votre action pour améliorer réellement cette situation?

- Culture scientifique : Elle n'est pas une priorité nationale. Sa diffusion dans les médias et vers le système scolaire est notoirement insuffisante. Pensez-vous pouvoir remédier à cette situation fâcheuse et comment?

- Impact sociétal : Nombre de questions sociétales majeures (climat, énergies, ondes électromagnétiques, OGM, recherche génétique, nanotechnologies...) sont très mal traitées en France, avec utilisation aveugle du principe de précaution et débats publics tronqués. Que ferez-vous pour résoudre ce problème préjudiciable à notre place dans le monde?

- Économie de la connaissance : Quelles seront vos priorités en matière d'éducation, en particulier dans les filières scientifiques et techniques? Quelle politique de l'emploi scientifique comptez-vous mettre en ouvre? Quelles seront vos thématiques prioritaires?

Nous vous remercions de l'attention que vous porterez à cette démarche et vous prions de bien vouloir agréer, Madame, Monsieur, l'expression de notre haute considération.

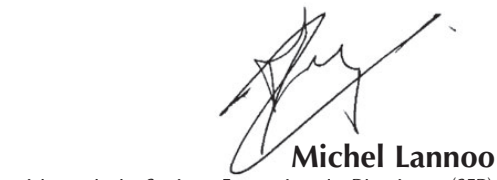

Président de la Société Française de Physique (SFP) Directeur de Recherche émérite au CNRS 